\title{
Left Ventricular Function and Cardiovascular Responses during Moderate Exercise; Investigation of the Exercise M-mode Echocardiography
}

\author{
Yasutomo Sakai ${ }^{1)}$, Natsuko Fukuhara ${ }^{2)}$, Tokiko Anzai ${ }^{3)}$, Shintaro Ueda ${ }^{4)}$ \\ ${ }^{1)}$ Department of Physical Therapy, Ibaraki Prefectural University of Health Sciences School of \\ Health Sciences, 4669-2 Ami, Ami-cho, Inashiki-gun, Ibaraki 300-03, Japan. \\ TEL +81 298-40-2219, FAX +81 298-40-2319, E-mail sakaiyasut@ipu.ac.jp. \\ ${ }^{2}$ Department of Physical Therapy, Tateyama Hospital \\ ${ }^{3)}$ Department of Physical Therapy, Sohsen Hospital \\ ${ }^{4}$ Doctor's Course in Medical Care-Welfare Engineering, Graduate School of Science and \\ Technology, Nihon University
}

\begin{abstract}
Abstsract. Using exercise echocardiography (M-mode method) with a supine-position leg ergometer, we examined cardiovascular responses and the contractile wall movement and pumping function of the left ventricle in healthy male subjects. Under a moderate exercise load equivalent to the optimum exercise intensity, it is necessary to fully understand changes in PRP and systolic blood pressure to accurately estimate myocardial oxygen demand. Decreases in the telediastolic and telesystolic volumes of the left ventricle, especially a marked decrease in the telesystolic volume, led to differences between the two volumes, resulting in an increased EF. There was a high correlation between changes in PRP and EF during exercise. Although exercise echocardiography requires complex measurement techniques, it is very useful in physiotherapy for risk control in cardiac rehabilitation.
\end{abstract}

Key words: Exercise echocardiography, Ejection fraction (EF), Healthy male subjects.

(This article was submitted Sep. 15, 1996, and was accepted Nov. 20, 1996)

\section{INTRODUCTION}

The contractile function of the left ventricle strongly correlates with the prognosis of ischemic heart disease, and is thus very useful as an index in the evaluation of the severity of the disease and the effects of therapy ${ }^{1)}$. Ischemic heart disease is diagnosed using tomographic echocardiography, cardiac pool scintigraphy, and left ventricular imaging. Among these methods, tomographic echocardiography, unlike the other methods, is a convenient and non-invasive physical examination that enables the simultaneous evaluation of the car- diac pumping function and systolic wall movement without the infliction of pain on patients. In recent years, exercise echocardiography ${ }^{2-5)}$ has been clinically used and has been suggested as a useful means for evaluating risk control in cardiac rehabilitation.

This study was performed as part of basic studies to examine the application of exercise echocardiography in physiotherapy. In this study, we examined the clinical usefulness of exercise echocardiography by using a supine-position leg ergometer as an exercise machine to compare the left ventricular ejection fraction $(\mathrm{EF})$, indicative of cardiac pumping function, with the product of the 
heartrate and systolic blood pressure (PRP), which is highly correlative with myocardial oxygen demand.

\section{METHODS}

The subjects of this study were five healthy males aged 21-34 (average 27.8+/- 5.1). The exercise machine was a leg ergometer (Aerobike 710, Combi Co., Ltd.) set for pedaling in the supine position. To prevent body movement of the subjects, the scapular and pelvic bands were fixed with belts. The exercise load was increased stepwise. The initial load was $30 \mathrm{~W}$, and was increased at increments of $30 \mathrm{~W}$ every three min. The exercise was stopped at $70 \%$ of the age-estimated maximum heart rate (estimative equation for young men $Y=201.7-0.583 X ; X=a g e)$. Heart rate (HR) was calculated from R-R intervals of ten beats before and after measurement, using an elec- trocardiograph (Cardiofax V ECAP 512, Nihon Kohden Corporation). Systolic blood pressure (SBP) was determined by auscultation of the radial artery. In tomographic echocardiography, the Mmode of the longitudinal section of the left ventricle was recorded (SSH-140A, TOSHIBA) from rest to the end of the exercise. Using the same apparatus, the telesystolic diameter of the left ventricle (Ds), telediastolic diameter of the left ventricle (Dd), and the left-ventricular ejection time (ET) were determined. Figure 1 shows the M mode of the longitudinal section of the left ventricle, Dd, and Ds. The determinations of HR and SBP and echocardiography were carried out at rest (after three minutes of rest) and every three minutes after the start of exercise (3, 6, 9, and $12 \mathrm{~min}$ after the start of exercise). Using data obtained in this way, the values of PRP $(\mathrm{HR} \times \mathrm{SBP}), \mathrm{EF}(\{1-$ $\left.\left.\mathrm{Ds}^{3}\right\} / \mathrm{Dd}^{3} \times 100\right), \operatorname{Vcf}(\{\mathrm{Dd}-\mathrm{Ds}\} / \mathrm{Dd} \times \mathrm{ET})$, and $\mathrm{EF}$ $\times$ Vcf were calculated.

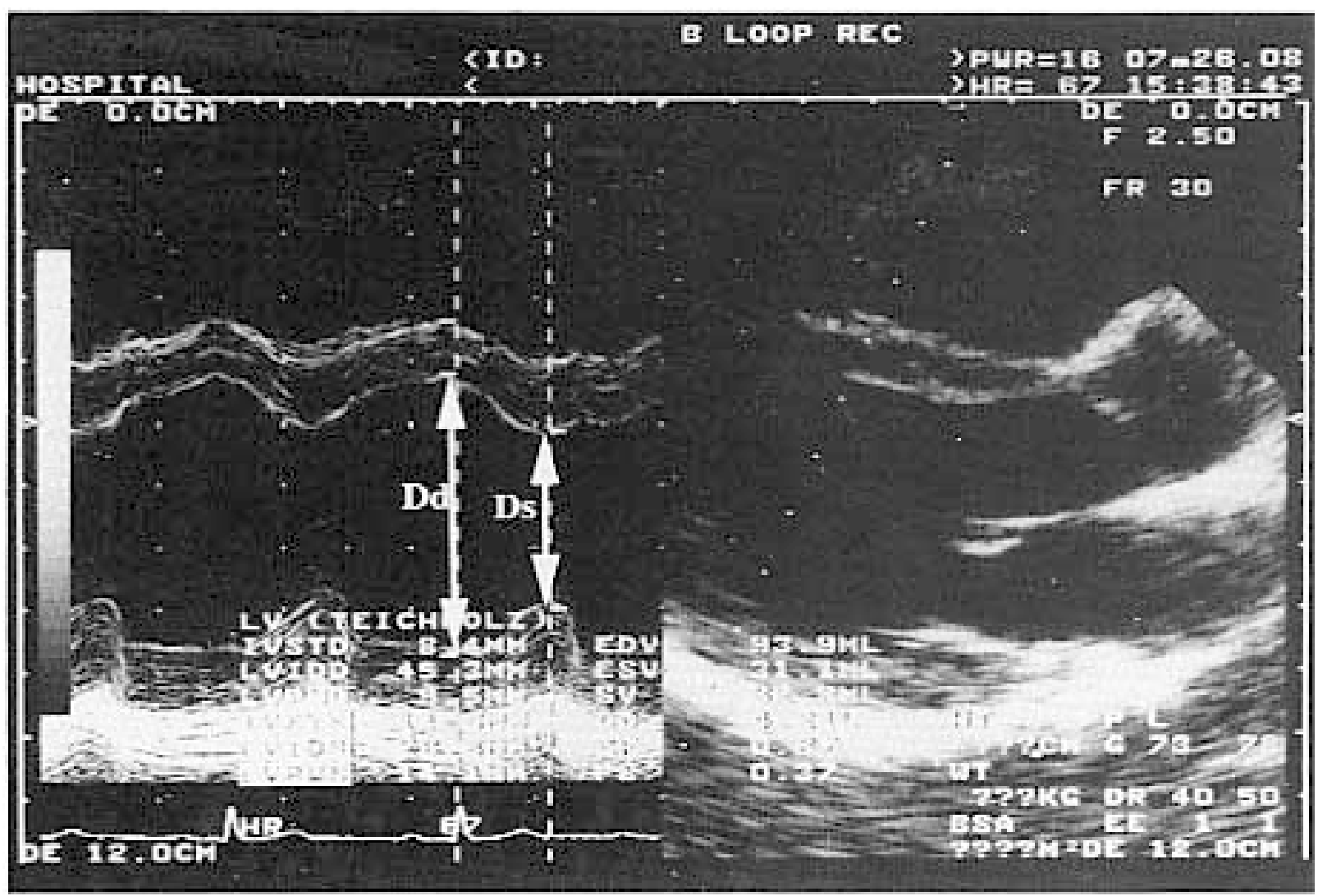

Fig. 1. Echocardiograph.

Left graph is a M mode of the longitudinal section of the left ventricle, Dd and Ds. Right graph is a tomographic echocardiograph of the left ventricle. 
All data were analyzed using Stat View software (J-4.5, Abucus). Changes in each parameter due to the gradually increased exercise load were analyzed at each loading stage using two-way ANOVA. The comparison of various parameters between rest and exercise was performed using the correlation analysis (in particular, partial correlation analysis).

\section{RESULTS}

All of the subjects attained $70 \%$ of the age-estimated maximum heart rate at the end of $120 \mathrm{~W}$ exercise load and completed the test. Figure 2 shows the parameters of cardiovascular responses (HR, \%HR, SBP, and PRP) during leg-ergometric exercise. Figures 3 and 4 show the parameters of left ventricular systolic wall movement (Dd, Ds, and $\mathrm{EF}$ ), and those of cardiac pumping function $(\mathrm{ET}, \mathrm{Vcf}$, and ET $\times \mathrm{Vcf})$, respectively. There were determined using leg-ergometric exercise echocardiography. At rest, the HR value was 69.80 +/- 8.78 beats/min, \%HR 37.4 +/- 4.6\%, SBP
$123.6+/-15.6 \mathrm{mmHg}$, PRP 8626.0 +/- 1475.5 beats $\cdot \mathrm{mmHg} / \mathrm{min}$, Dd 59.6 +/- $6.6 \mathrm{~mm}$, Ds $36.3+/-$ $5.0 \mathrm{~mm}$, EF $77.5+/-3.0 \%$, ET $0.36+/-0.04 \mathrm{sec}$, Vcf $1.01+/-0.09 \mathrm{sirc} / \mathrm{sec}$, and ET $\times$ Vcf $84.53+/-$ 7.52 sirc.

The ANOVA revealed that all parameters excluding Dd changed significantly throughout the study $(\mathrm{p}<0.002)$. With increasing exercise loads, the HR, \%HR, SBP, EF, Vcf, and EF $\times$ Vcf values tended to increase, while the Dd, Ds, and ET values tended to decrease.

The relationships between the decrease in the various parameters with increasing exercise loads were analyzed using correlation analysis and partial correlation analysis, excluding double correlation. These results are shown in Table 1. The parameters were divided into two groups: cardiovascular responses, and left ventricular systolic wall movement and cardiac pumping function. The parameters were compared in each group. In the former group (cardiovascular responses), there were significant partial correlations between PRP and SBP and between HR and SBP, and PRP

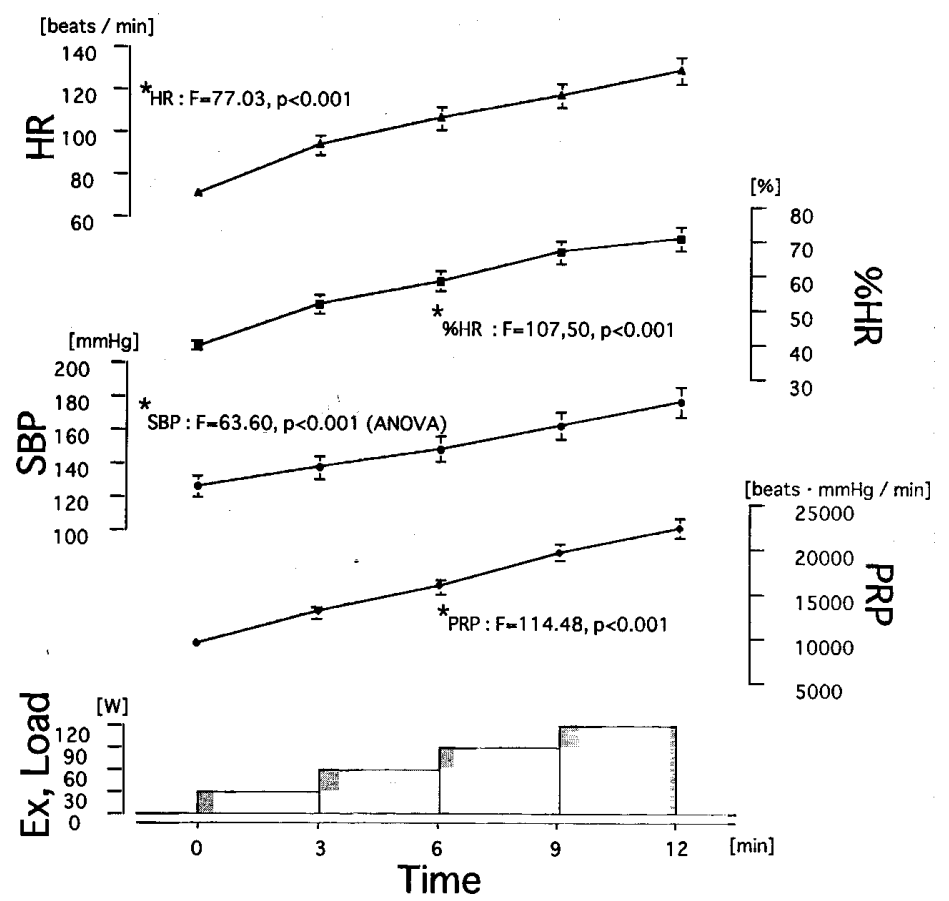

Fig. 2. Effect of exercise stress (leg ergometer) on general cardiovascular response indices; Heart rate (HR), \% Heart rate (\%HR), Systolic blood pressure (SBP), Pressure rate poroducts (PRP). Values ( $\emptyset$ ) are means \pm standard deviation; the statistical significance is $*(\mathrm{p}<0.01)$ with ANOVA. 

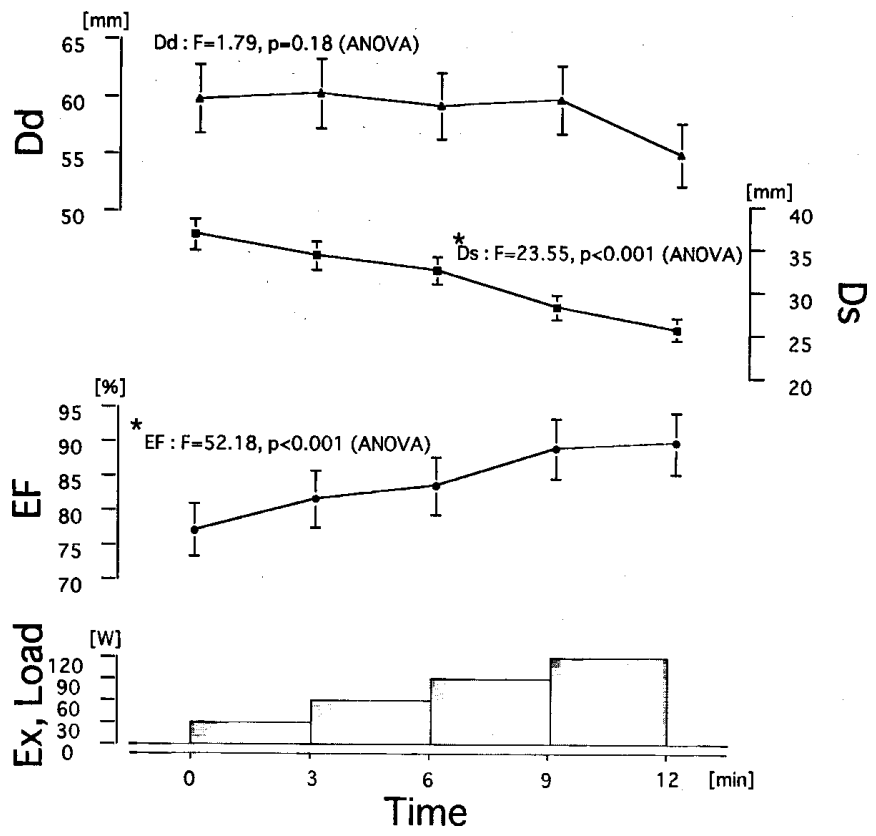

Fig. 3. Echocardiographic left ventricular wall-kinesis and pumping-function parameters during exercise stress (leg ergometer); End-systolic diameter of Left ventricle (Ds), End-diastolic diameter of Left ventricle (Dd), Ejection fraction (EF). Values $(\Phi)$ are means \pm standard deviation; the statistical significance is $*(\mathrm{p}<0.01)$ with ANOVA.
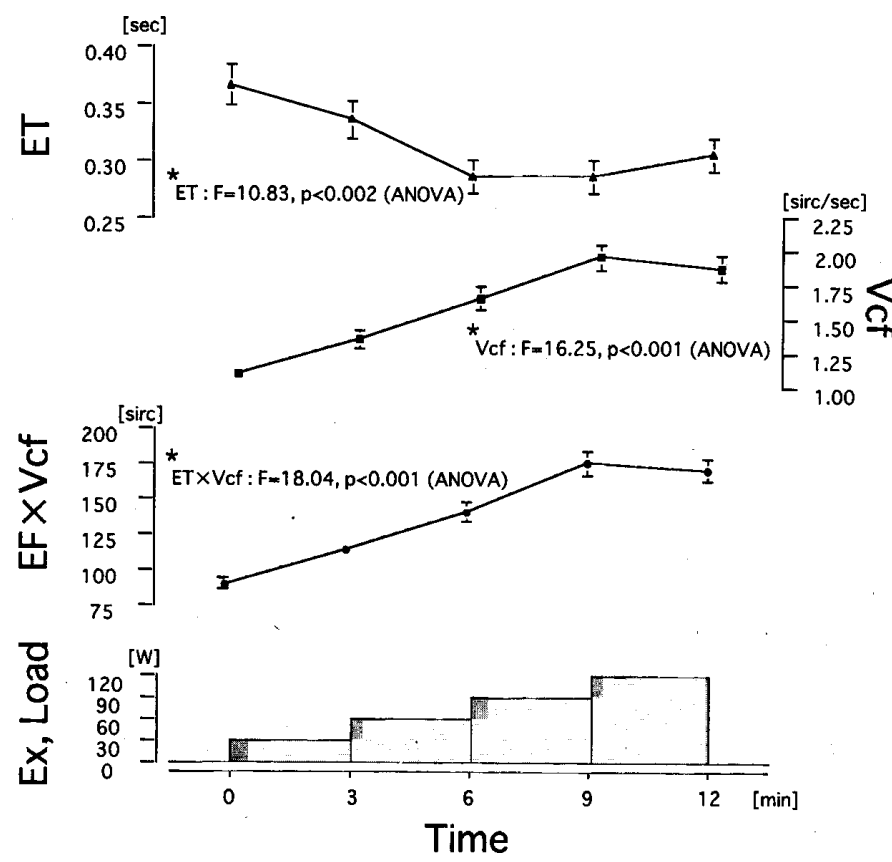

Fig. 4. Echocardiographic left ventricular wall-kinesis and pumping-function parameters during exercise stress (leg ergometer); velocity of circumferential fiber shortening $(\mathrm{Vcf}), \mathrm{EF} \times \mathrm{Vc}$. Values $(\underline{\mathbf{q}})$ are means \pm standard deviation; the statistical significance is $*(\mathrm{p}<0.01)$ with ANOVA. 


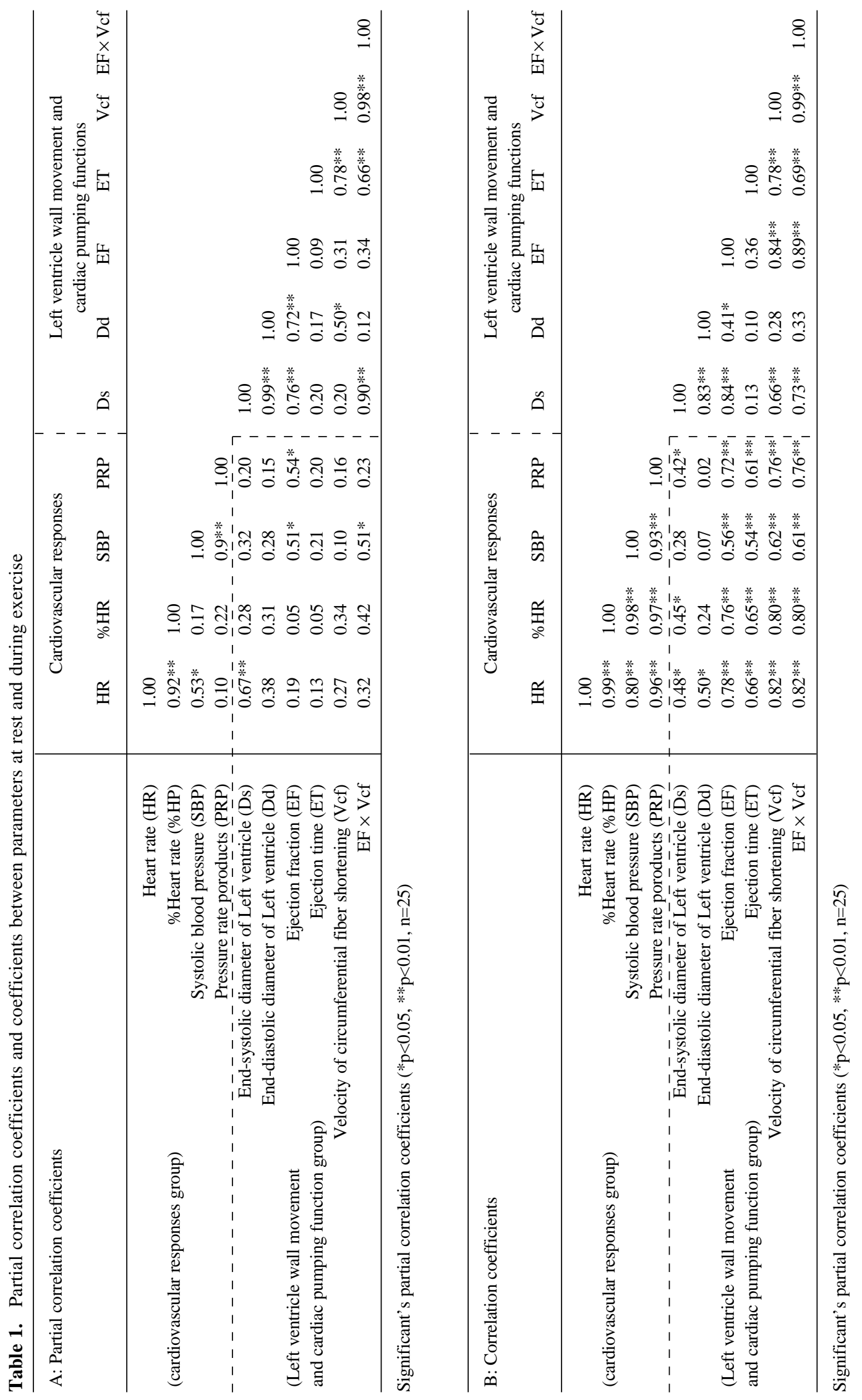


tended to increase as a result of its dependence on SBP ( $r=0.93$ ). In the latter group (left ventricular systolic wall movement and cardiac pumping function), there were significant partial correlations between Dd and Ds, EF and Ds, EF and Dd, and Ds and EF $\times$ Vcf. Both Ds and Dd decreased $(r=0.83)$, though the degree of decrease was higher for Ds ( $r=0.84)$ than for Dd ( $r=0.41)$. As the difference between Ds and Dd increased, the EF increased. In comparing the parameters of the two groups, significant partial correlations between HR and Ds, EF and SBP, EF and PRP, and SBP and $\mathrm{EF} \times \mathrm{Vcf}$ were identified. In addition, EF increased as a result of its dependence on SBP $(r=0.56)$, rather than on PRP ( $\mathrm{r}=0.72$ ), which is thought to be indicative of myocardial oxygen demand.

\section{DISCUSSION}

Myocardial oxygen demand is determined primarily by (1) myocardial contractility, (2) ventricular tension, and (3) heartrate ${ }^{6}$.

The present experiments showed that the PRP value, which is the product of the heartrate and systolic blood pressure, correlated highly with the left ventricular ejection fraction. These experiments also revealed that changes in PRP were induced by changes in systolic blood pressure rather than heartrate. This indicates that increased systolic blood pressure can predict a concurrent increase in myocardial oxygen demand. In other words, to estimate myocardial oxygen demand, it is important to understand changes in systolic blood pressure. In the experiments, echocardiography was performed by the M-mode method, and Ds, Dd, and $\mathrm{EF}$, primarily, were analyzed. As the exercise load was increased, Ds and Dd reduced. The degree of the reduction was higher for Ds than for $\mathrm{Dd}$, and the increased difference between Ds and Dd led to an increase in the EF value. That is, the moderate exercise load caused the telediastolic and telesystolic volumes of the left ventricle to decrease, while the telesystolic volume decreased even more markedly. Thus, there was found to be an increase in the ejection fraction, which is given by dividing the difference between the two volumes by the telediastolic volume. Sharma et al. ${ }^{3)}$ obtained similar results using left ventricular imaging, and also reported that exercise load increased the telediastolic and telesystolic volumes of the left ventricle in cases of coronary artery disease, thus decreasing the left ventricular ejection fraction.

In evaluating myocardial infarction using traditional exercise electrocardiography, the local wall movement cannot be estimated and evidence of new ischemia cannot be found using an electrocardiogram during exercise in cases of myocardial infarction with existing cardiographic abnormality. In contrast, exercise echocardiography allows the separate observation of the wall movements of uninfarcted and infarcted areas during exercise ${ }^{4}$. In the estimation of coronary arterial lesions, exercise echocardiography is slightly higher in sensitivity than exercise electrocardiography, and has been reported to be very helpful in examining patients with cardiographic abnormality even at rest, patients in whom cardiographic abnormality is induced by standing or hyperapnea, and patients with changes in non-specific ST-T waves due to cardiomegaly7). In these areas, exercise echocardiography is clinically useful and effective in controlling the risk of coronary artery diseases. With respect to precision, the results of echocardiography are at least $85 \%$ consistent with those of heart pool scintigraphy. It has been reported that there are no difficulties with echocardiography measurement techniques, which have long been considered the major disadvantage of echocardiography, and no problems with it's reproducibility ${ }^{2}$. There have also been many reports on the trial use of this method as a routine study5). Based on these reports and after comparing the results of this study with those of other studies, we judged exercise echocardiography to be a credible test method.

This method could also have useful clinical applications in physiotherapy for risk control in cardiac rehabilitation. We believe that in the future physiotherapy will require clinical data obtained from a combination of exercise echocardiography and exercise electrocardiography.

\section{REFERENCES}

1) Bhatnagar SK, et al: The role of prehospital discharge two-dimensional echocardiography in determining the prognosis of survivors of first myocardial infarction. American Heart Journal 109 (3 Pt 1): 472-477, 1985.

2) Yamasawa $M$, et al: Clinical use of exercise twodimensional echocardiography for evaluating effects of percutaneous transfunctional coranary angioplasty and coronary artery bypass graft. Jpn J Med Ultrason- 
ics 18 (3): 238-247, 1991.

3) Sharma B, et al: Left ventricular angiography on exercise; A new method of assessing left ventricular function in ischaemic heart disease. British Heart Journal 38 (1): 59-70, 1976.

4) Yamasawa M, et al: Prediction of coronary artery lesions by two-dimensional echocardiography at rest and during exercise: in patients with left anterior descending artery lesion. Nippon Naika Gakkai Zasshi, Journal of Japanese Society of Internal Medicine 78: 9-14, 1989.

5) Kuroda T, et al: Prognostic value of peak exercise 2- dechocardiographic analysis after acute myocardiographic analysis after acute myocardial infarction; a prospective 5-year follow-up study. Circulation 86 (suppl I): 790, 1992.

6) Parmley WW, et al: Medical therapy of angina pectoris. Angiology 28: 865-872, 1977.

7) Folland ED: Assessment of left ventricular ejection fraction and volumes by real-time, two-dimensional echocardiography. A comparison of cineangiographic and radionuclide techniques. Circulation 60: 760 766, 1979. 